\title{
Evolution and conservation of polycomb repressive complex 1 core components and putative associated factors in the green lineage
}

Yong Huang ${ }^{1,2,3}$, Ling Jiang ${ }^{1,2,3}$, Bo-Yu Liu ${ }^{1,2,3}$, Cheng-Fang Tan ${ }^{1,2,3}$, Dong-Hong Chen ${ }^{4}$, Wen-Hui Shen ${ }^{2,5}$ and Ying Ruan ${ }^{1,2,3^{*}}$

\begin{abstract}
Background: Polycomb group (PcG) proteins play important roles in animal and plant development and stress response. Polycomb repressive complex 1 (PRC1) and PRC2 are the key epigenetic regulators of gene expression, and are involved in almost all developmental stages. PRC1 catalyzes H2A monoubiquitination resulting in transcriptional silencing or activation. The PRC1 components in the green lineage were identified and evolution and conservation was analyzed by bioinformatics techniques. RING Finger Protein 1 (RING1), B lymphoma Mo-MLV insertion region 1 homolog (BMI1), Like Heterochromatin Protein 1 (LHP1) and Embryonic Flower 1 (EMF1) are the PRC1 core components and Vernalization 1 (VRN1), VP1/ABI3-Like 1/2/3 (VAL1/2/3), Alfin-like 1-7 (AL1-7), Inhibitor of growth 1/2 (ING1/2), and Early Bolting in Short Days (EBS) / Short Life (SHL) are the associated factors.

Results: Each PRC1 subunit possesses special domain organizations, such as RING and the ring finger and WD40associated ubiquitin-like (RAWUL) domains for RING1 and BMI1, chromatin organization modifier (CHROMO) and chromo shadow (ChSh) domains for LHP1, one or two B3 DNA binding domain(s) for VRN1, B3 and zf-CW domains for VAL1/2/3, Alfin and Plant HomeoDomain (PHD) domains for AL1-7, ING and PHD domains for ING1/2, Bromoadjacent homology (BAT) and PHD domains for EBS/SHL. Six new motifs are uncovered in EMF1.

The PRC1 core components RING1 and BMI1, and the associated factors VAL1/2/3, AL1-7, ING1/2, and EBS/SHL exist from alga to higher plants, whereas LHP1 only occurs in higher plants. EMF1 and VRN1 are present only in eudicots. PRC1 components undergo duplication in the plant evolution. Most of plants carry the homologous core component LHP1, the associated factor EMF1, and several homologs in RING1, BMI1, VRN1, AL1-7, ING1/2/3, and EBS/SHL. Cabbage, cotton, poplar, orange and maize often exhibit more gene copies than other species. Domain organization analysis shows that duplicated gene functions may be of diverse.
\end{abstract}

Conclusions: The PRC1 core components RING1 and BMI1, and the associated factors VAL1/2/3, AL1-7, ING1/2, and EBS/SHL originate from algae. The core component LHP1 is from moss and the associated factors EMF1 and VRN1 are from dicotyledon. PRC1 components are of functional redundancy and diversity in evolution.

Keywords: Polycomb, PRC1, Phylogenetic analysis, Domain organization, Evolution

\footnotetext{
* Correspondence: yingruan@hotmail.com

${ }^{1}$ Key Laboratory of Crop Epigenetic Regulation and Development in Hunan

Province, Hunan Agricultural University, Changsha 410128, China

${ }^{2}$ International Associated Laboratory of CNRS-FU-HAU on Plant Epigenome

Research, Hunan Agricultural University, Changsha 410128, China

Full list of author information is available at the end of the article
}

(c) The Author(s). 2019 Open Access This article is distributed under the terms of the Creative Commons Attribution 4.0 International License (http://creativecommons.org/licenses/by/4.0/), which permits unrestricted use, distribution, and reproduction in any medium, provided you give appropriate credit to the original author(s) and the source, provide a link to the Creative Commons license, and indicate if changes were made. The Creative Commons Public Domain Dedication waiver (http://creativecommons.org/publicdomain/zero/1.0/) applies to the data made available in this article, unless otherwise stated. 


\section{Background}

Polycomb-group (PcG) proteins play essential roles in epigenetically repressing or activating genes transcription in animals and plants, and are implicated in stable and heritable transcriptional silencing or activating of target genes during organism development [1-5]. PcG proteins are evolutionarily conserved and are involved in various aspects of plant development, such as the timing of flowering and seed development [3, 6-8], and response to abiotic and biotic stresses [9]. In contrast with animal PcG proteins, which are involved in maintaining pluripotency and preventing cell differentiation, plant PcG proteins are required to promote cell differentiation by suppressing embryonic development [10].

Polycomb proteins can form at least three distinct complexes in metazoan, namely polycomb repressive complex 1 (PRC1), PRC2 and polycomb-like PRC2 (Pcl-PRC2) [11]. PRC1 and PRC2 complexes were found earlier than PclPRC2 [12-14]. PRC2 is attributed to the trimethylation of lysine-27 on histone H3 (H3K27me3) [15]. Pcl-PRC2 is required to generate high levels of $\mathrm{H} 3 \mathrm{~K} 27 \mathrm{me}$ at polycomb target genes [16]. PRC1 catalyzes $\mathrm{H} 2 \mathrm{~A}$ monoubiquitination (H2Aub) and causes transcriptional silencing after histone H3K27me induced by PRC2 or via an unknown mechanism [17].

PRC1 was previously believed to be the major determinant in silencing genes via histones $\mathrm{H} 2 \mathrm{~A}$ ubiquitination [18]. PRC1 also facilitates transcription of many genes [19]. PRC1 maintains gene silencing through interfering with recruited mediators to inhibit activated RNA polymerase II preinitiation complex assembly [20]. Histone H3K27me induced by $\mathrm{PRC} 2$ provides a binding site for the N-terminal chromodomain of the PRC1 Pc subunit; furthermore, PRC1 catalyzes H2Aub via its RING1a/b subunit, the Really Interesting New Gene (RING) domain of which exhibits E3 ubiquitin ligase activity [18, 20, 21]. BMI1 and RING1a positively regulate $\mathrm{H} 2 \mathrm{~A}$ ubiquitylation, resulting Hox gene silencing [22]; $\mathrm{BAH}$ domain proteins EBS/SHL-EMF1 complex mediates genome-wide transcriptional repression by effecting H3K27me3 [23]. PRC1 directly influences the transcription of active genes. PRC1 subunit mutations alter the phosphorylation of RNA polymerase II and occupancy by the Spt5 pausing-elongation factor at most active genes [19]. RING1a and BMI1 also activate genes via chromatin-associated protein Ubiquitination $[24,25]$.

Drosophila core PRC1 is composed of four subunits, Polycomb (Pc), Posterior sex combs (Psc), Polyhomeotic $(\mathrm{Ph})$ and Sex combs extra (Sce), but only Pc, Psc and Sce are found in plants $[2,26]$. In plants, PRC1 was thought to be absent until Arabidopsis RING finger proteins including RING1a/b and BMI1a/b/c have been characterized. Plant PRC1 participates in multiple processes during vegetative and reproductive development, such as the control of stem cell fate determinacy, the prevention of vegetative-to-embryogenesis conversion, flowering timing, floral organ number and identity, and seed development [27, 28]. Arabidopsis PRC1 complex comprises of three core components, ring finger protein 1 (RING1a/1b), B lymphoma Mo-MLV insertion region 1 homolog $(\mathrm{BMI} 1 \mathrm{a} / \mathrm{1b} / 1 \mathrm{c})$ and like heterochromatin protein 1(LHP1), and six associated components, Embryonic Flower 1 (EMF1), Vernalization 1 (VRN1), VP1/ ABI3-Like 1/2/3 (VAL1/2/3), Alfin-like 1-7 (AL1-7), Inhibitor of growth (ING1/2), and Early Bolting in Short Days /Short Life (EBS/SHL) (Fig. 1, Additional file 1). PRC1 complexes, including AtRING1/AtBMI-PRC1, EMF1-PRC1 and PRC2-H3K4 demethylase-PRC1, have been proposed [29]. The two prominent roles of PRC1 complex are: as a reader of H3K27me3, LHP1 with specific H3K27me3 binding depending on the chromatin organization modifier (CHROMO) domain; as a writer of H2Aub1, RING1 and BMI1 providing ubiquitin ligase activity relied on RING domains [19, 30]. EBS and SHL are bivalent $\mathrm{H} 3 \mathrm{~K} 27 \mathrm{me} 3$ and $\mathrm{H} 3 \mathrm{~K} 4 \mathrm{me} 3$ readers [31]. However AtRING1s and AtBMI1s are required for H2AK119ub, but not for PRC2 induced H3K27me3 [29].

The PRC1 subunit EMF1 cooperates with PRC2 to repress key regulators in Arabidopsis development [32]; however EMF1 and VRN1 exist in dicotyledon species [27]. VRN1 and VAL1/2/3 are plant-specific components of PRC1 [29]. VAL1 and PRC1 form a complex via interaction with AtBMI1a/1b [17, 33]. The VAL1-PRC1 complex maintenances differentiated state, such as seed maturation, through catalyzing $\mathrm{H} 2 \mathrm{Aub}$ by specifically binding to gene promoters [17, 34]. PRC1 also acts as both a reader of $\mathrm{H} 3 \mathrm{~K} 4 \mathrm{me} 3$ and a writer of $\mathrm{H} 2 \mathrm{Aub} 1$ in establishing a stable repression chromatin state [35]. AL proteins are novel interactors of the PRC1 core components AtBMI1a and AtRING1b [36]. AL proteins target PRC1 to form AL PHD-PRC1 complexes with active chromatin for transitioning from H3K4me3 to H2Aub1/ H3K27me3 to establishing gene silencing [35]. Epigenetic regulatory protein ING proteins are characterized by a nuclear localization sequence and a high affinity to $\mathrm{H} 3 \mathrm{~K} 4 \mathrm{me} 3$; however the role of these proteins as associated factors of PRC1 remains unclear [37]. Lee et al. showed that ING and AL are nuclear proteins involved in chromatin regulation through binding to $\mathrm{H} 3 \mathrm{~K} 4 \mathrm{me} 3 / 2$ [38]. ING1 is regulated in response to activity by binding to a site upstream of the Ppp3r1 transcription start site [39]. EBS and SHL, the plant-unique BAH-domain containing proteins, implement Polycomb silencing or activating by forming a complex with EMF1, and function as such as floral phase transition [23, 31].

PRC1 are highly divergent between animals and plants. Core components and associated subunits of PRC1 form functional complex. The PRC1 core components RING- 


\begin{tabular}{|c|c|c|c|c|c|c|c|c|c|c|c|}
\hline & & & $\begin{array}{l}\text { RING } \\
1 \mathrm{a} / 1 \mathrm{~b}\end{array}$ & $\begin{array}{c}\mathrm{BMI} \\
1 \mathrm{a} / 1 \mathrm{~b} / 1 \mathrm{c}\end{array}$ & LHP1 & EMF1 & VRN1 & $\begin{array}{l}\text { VAL } \\
1 / 2 / 3\end{array}$ & $\begin{array}{l}\text { AL } \\
1-7\end{array}$ & $\begin{array}{r}\text { ING } \\
1 / 2 \\
\end{array}$ & $\begin{array}{l}\text { EBS } \\
\text { ISHL }\end{array}$ \\
\hline & & Arabidopsis thaliana (At) & 2 & 3 & 1 & 1 & 5 & 3 & 7 & 2 & 2 \\
\hline & & Lapsella grandiflora $(\mathrm{Cg})$ & 2 & 3 & 1 & 1 & 3 & 3 & 6 & 2 & 3 \\
\hline & & Boechera stricta (Bs) & 2 & 3 & 1 & 1 & 4 & 3 & 7 & 2 & 3 \\
\hline & & Brassica rapa $(\mathrm{Br})$ & 3 & 3 & 3 & 4 & 7 & 5 & 15 & 2 & 4 \\
\hline & & Eutrema salsugineum (Es) & 2 & 3 & 1 & 2 & 4 & 3 & 6 & 2 & 3 \\
\hline & & - Gossypium raimondii (Gr) & 2 & 5 & 1 & 2 & 13 & 6 & 12 & 3 & 7 \\
\hline & & - Citrus sinensis (Or) & 2 & 3 & 1 & 1 & 11 & 3 & 3 & 2 & 2 \\
\hline & & Populus trichocarpa (Pt) & 4 & 5 & 2 & 1 & 5 & 6 & 9 & 3 & 6 \\
\hline & & $\lceil$ Fragaria vesca $(\mathrm{Fv})$ & 2 & 2 & 1 & 1 & 9 & 3 & 4 & 2 & 2 \\
\hline & Magnoliophyta- & $\mathrm{L}_{\text {Cucumis sativus (Cs) }}$ & 2 & 3 & 2 & 2 & 9 & 3 & 5 & 2 & 3 \\
\hline & & -Zea mays $(\mathrm{Zm})$ & 4 & 6 & 1 & 0 & 0 & 3 & 16 & 2 & 4 \\
\hline & & પ Oryza sativa (Os) & 2 & 3 & 1 & 0 & 0 & 2 & 8 & 2 & 4 \\
\hline & & Brachypodium distachyon (Bd) & 2 & 4 & 1 & 0 & 0 & 2 & 9 & 3 & 4 \\
\hline & LLycopodiophyta - & Selaginella moellendorffii (Sm) & 1 & 2 & 1 & 0 & 0 & 1 & 3 & 2 & 2 \\
\hline Archaeplastida- & L Bryophyta & Physcomitrella patens (Pp) & 2 & 2 & 1 & 0 & 0 & 5 & 7 & 2 & 6 \\
\hline & & Chlamydomonas reinhardtii $(\mathrm{Cr})$ & 1 & 0 & 0 & 0 & 0 & 1 & 1 & 2 & 1 \\
\hline & LChlorophyta & L volvox carteri (Vc) & 1 & 1 & 0 & 0 & 0 & 1 & 1 & 2 & 1 \\
\hline & & LOstreococcus lucimarinus (OI) & 0 & 0 & 0 & 0 & 0 & 0 & 1 & 1 & 1 \\
\hline
\end{tabular}

Fig. 1 PRC1 proteins in representative plants. Numbers show the copy numbers of homologous proteins in each plant. Data were derived from Phytozome 12.0 (http://www.phytozome.net) and Brassica Database (http://brassicadb.org/brad/)

finger proteins (RING1 and BMI1) and LHP1 exist in the ancestor of seed plants, whereas EMF1, VRN1, and EBS/SHL have been recently identified [27, 31, 40, 41]. RING1, BMI1 and LHP1 proteins have originated from mosses [42]. However the evolution of PRC1 associated factors, VRN1, EBS/SHL, VAL, AL and ING, remains unclear. Core components and associated of PRC1 are distributed in different plant species. The rapidly accumulating data of sequenced plant genomes allows to conduct systematic studies on plant gene evolution and functional differentiation. Therefore, this study aims to analyze the evolution and conservation of PRC1 core components and newfound associated factors, especially domain organizations and genes duplication, in the green lineage from lower to higher plants. The results would provide information for understand the evolution and conservation of plant PCR1.

\section{Results}

\section{PRC1 core component: RING1}

PRC1 RING finger proteins are composed of the two clades (Additional file 2), RING1 and BMI1, both of which are characterized by a conserved combination of RING and Ring-finger And WD40 associated Ubiquitin-Like (RAWUL) domains (Figs. 2 and 3). The ubiquitin ligase activity of PRC1 complex writers relies on their RINGdomain [27]. In animals, RING1b is a key H2Aub writer, whereas RING1a plays minor roles. BMI1 exhibits no E3 ligase activity but can stabilize and enhance RING1b functions [43, 44]. In Arabidopsis, both AtRING1a/b and
AtBMI1a/b/c families can catalyze H2Aub. At the vegetative stage, AtRING1a/1b can repress vegetative-toembryonic transition and ectopic meristem formation mainly via suppressing the mis-expression of embryo master regulators and stem cell regulators, respectively [45, 46]. At the reproductive stage, the Arabidopsis double mutant ring1a;ring $1 b$ exhibits exceedingly high numbers of floral organs, and strong phenotypes displaying dramatic swelling gynoecium and completely sterile [45]. Both AtRING1a and AtRING1b can control the maintenance of floral stem cells and proper carpel development by repressing KNOX-I expression [42]. RING1a/b mutation can cause early vegetative phase transition by regulating the H2Aub status at the SPL locus [47].

In a phylogenetic tree, plant RING1 proteins can be divided into three branches: seed plant (Group-I), moss-fern (Group-II), and algae (Group-III, Fig. 2). The phylogenetic relationship of RING1 homologs is consistent with plant evolution. RING1 has undergone one and two duplications in eudicot and monocot ancestors, respectively [40]. Most RING1 proteins exhibit two copies in each species, but PtRING1 and ZmRING1 occur in four copies, and BrRING1 present in three copies. However, the duplication event may occur after the separation of monocotyledons and dicotyledons. RING1 proteins in eudicots display similar domain organizations (Fig. 2). RING1 proteins in monocots are represented only by Poaceae, displaying few variable domain organizations. The typical RING domain, POU, which is a bipartite DNA binding domain, and Ras Exchanger Motif are also found in 


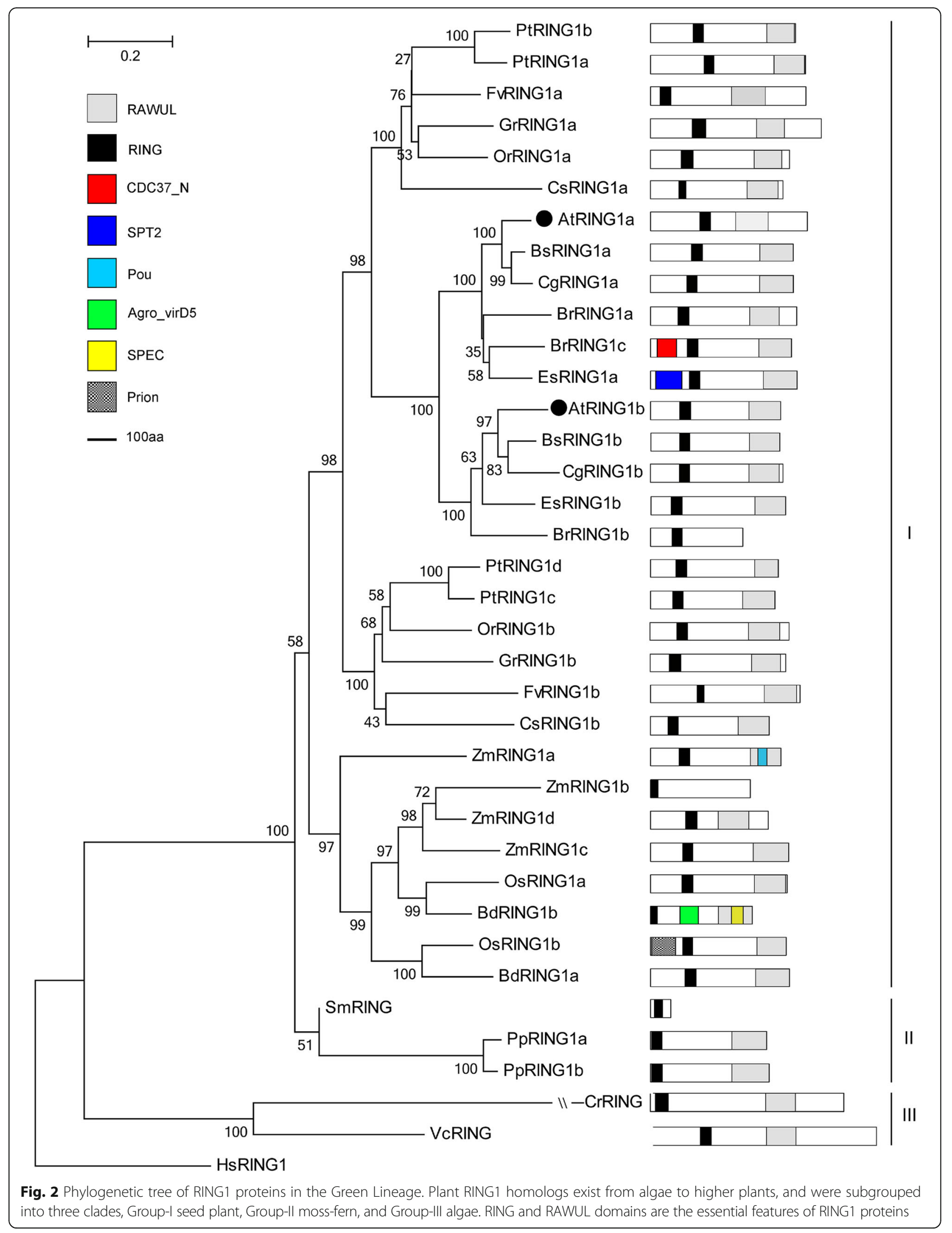




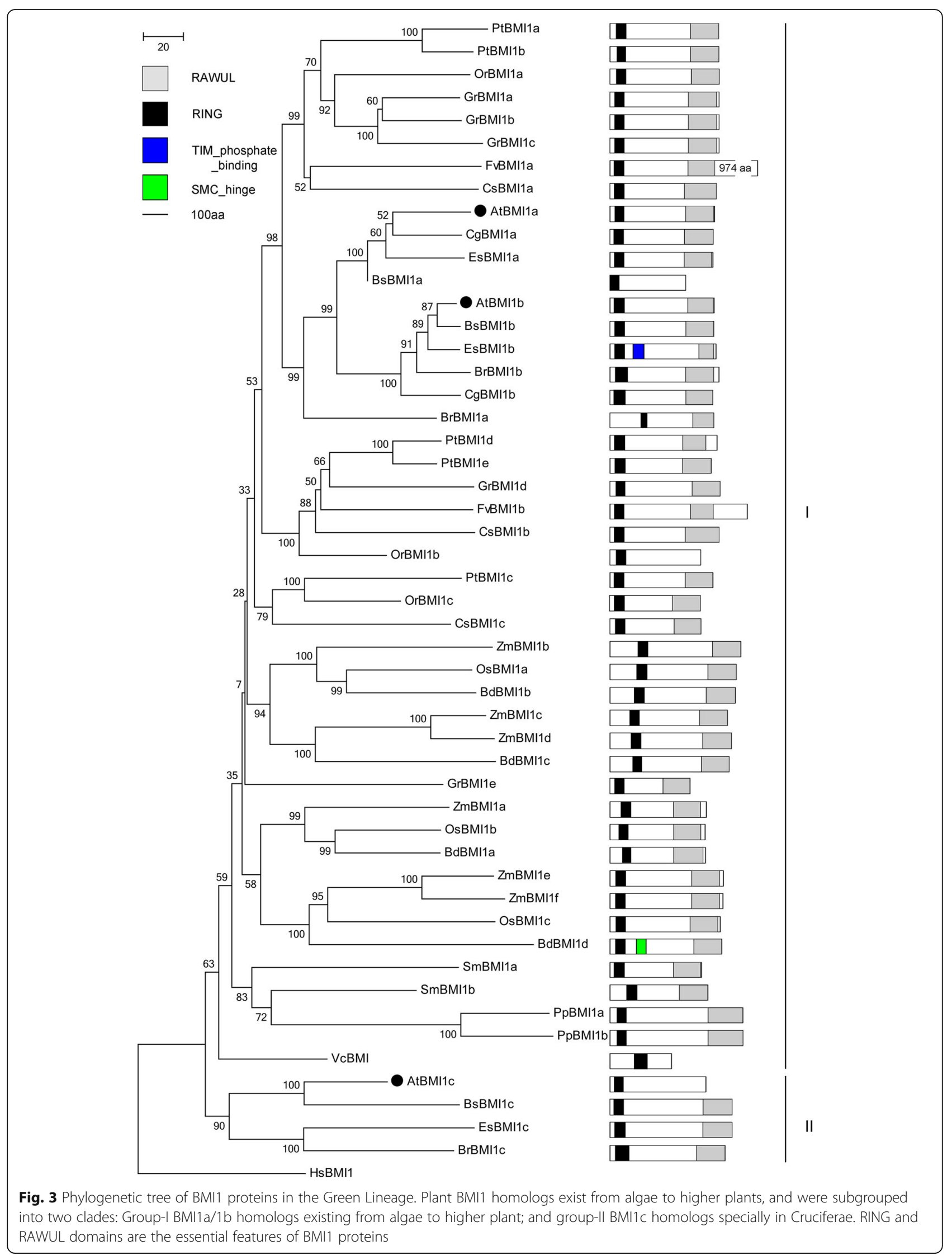


ZmRING1a, Agrobacterium VirD5 protein, and Spectrin repeats domains display in BdRING1b. Prion domain occurs in OsRING1b. Intriguingly, the POU domain was first identified in plants. Group-II exists in fern and Physcomitrella patens, whereas Group-III is present in two algae. However, both of these groups are well conserved in domain organizations.

\section{PRC1 core component: BMI1}

The three BMI1-like proteins, AtBMI1a, AtBMI1b, and AtBMI1c exist in Arabidopsis [48]. BMI1 deficiency (atbmila;atbmi1b double mutant) causes an embryonic-like structure at the vegetative stage and a high number of floral organs in the reproductive stage, a feature similarly found in the ring1a;ring $1 b$ double mutant $[45,46,49]$. Similar to RING1 proteins, BMI1a/1b functions as PRC1 writers for H2Aub, coordinating with PRC2-mediated H3K27me3 to maintain cell identity [50]. AtBMI1a/1b functions as an E3 ubiquitin ligases, and is involved in drought-response [51]. MIR156A and MIR156C are also target genes of AtBMI1, regulating vegetable to productive development transition [28]. Notably, AtBMI1c acts as an imprinted gene that expresses maternally allele in the endosperm but biallelically in the stamen [49]. BMI1 proteins can be identified in all plants, and in alga Volvox carteri, but not in the algae Ostreococcus lucimarinus or Chlamydomonas reinhardtii; furthermore, BMI1 are divided into two groups, namely BMI1a/1b and BMI1c homologs (Fig. 3). All BMI1s contain highly conserved RING and RAWUL domains except for BsBMI1a, PtBMI1d, and OrBMI1b, OrBMI1b lacks RAWUL domain. The sequence length of BMI1s usually occurs between 350 and 550 aa, but FvBMI1c comprises 974 aa residues with an overlong C-terminus. Dicotyledon contains three copies of BMI1 except for poplar and cotton five copies, and orange two copies. All BMI1a/1b proteins show similar domain organizations, but ThBMIlb harbors another TIMphosphate-binding motif adjacent to the RING domain; furthermore, BdBMI1d owns a Structural Maintenance of Chromosomes (SMC) proteins Flexible hinge motif, which is responsible for DNA dimerization and as an essential determinant of dynamic SMC-DNA interactions [52]. Highly conserved AtBMI1c and its homologs are only found in Crucifera (Fig. 3).

The RAWUL domain was first identified in the PRC1 RING finger proteins, RING1 and BMI1 families, and is conserved in plant and worm [48]. The RAWUL domain may be involved in epigenetic regulation by binding to PRC1 or other factors. In mammals, RAWUL has been shown to bind to $\mathrm{Ph}$ homologs though this phenomenon has not been confirmed to date. Thus, the RAWUL domain may bind to other proteins involved in histone ubiquitination. Sanchez-Pulido et al. suggested that some other proteins demonstrate PRC1 histone ubiquitination functions
[48]. Arabidopsis HTA10 displays the conserved PKKT consensus sequence [53]. Maize ubiquitinated H2A may be involved in H2A ubiquitination [54]. Grain RAWUL protein Gnp4/LAX2 regulates grain length via the auxin signaling pathway by interfering with OsIAA3-OsARF25 [55]. The RAWUL domain can form a protein-protein interaction module with the PAL domain of AL6 N-terminus, which is an associated factor of PRC1 $[36,48,56]$.

The RAWUL domain is not highly conserved between animals and plants. However, a sequence alignment analysis of RING1a/1b homologs shows that the domains are considerably conserved from lower to higher plants, and BMI1a/1b/1c lacks $\beta 5$. The RING proteins (BrRING1b, ZmRING1b and SmRING) and the BMI1 proteins (AtBMI1c, BsBMI1a, OrBMI1b and VcBMI) do not contain RAWUL domains (Figs. 2 and 3, Additional file 3) . RING and RAWUL domain are possibly be special domains for RING1 and BMI1 families.

\section{PRC1 Core component: LHP1}

In Arabidopsis, LHP1, an activator and a repressor of transcription, is first identified as a Drodophila Heterochromatin-associated Protein 1 (HP1) homolog that binds to H3K27m 3 markers established by PRC2 and catalyzes monoubiquitination at lysine 119 of histone H2A [57]. LHP1 may be an analogous role to the fly Pc in a PRC1-like complex [36]. LHP1 contains two typical domains, Chromatin Organization Modifier (CHROMO) domain which is essential for H3K27me3 binding specificity [10], and Chromo Shadow (ChSh) domain [58]. In contrast with its animal counterpart, LHP1 is predominantly located within the euchromatin [59]. The localization and retention of Fern LHP1 are controlled by distinct domains, and its retention at the nucleolus and chromocenters is conferred by the ChSh domain [60]. P. patens PpLHP1 interacts with PpCMT through their chromo domains [61]. As a PRC1 reader in plants [27], LHP1 controls multiple developmental pathways related to organ development, cell size, and vegetative to reproductive phase transitions [57, 62].

LHP1 homologs also undergo plant evolution. Aside from distinguished $\mathrm{CHROMO}$ and $\mathrm{ChSh}$ domains, some LHPs contain other distinct motifs (Fig. 4). For example, poplar LHP1s have an additional CDC37 domain in their $\mathrm{N}$-terminus, and AtLHP1 comprise an additional B5 domain found in phenylalanine-tRNA synthetase $\beta$ subunits. OsLHP1 consists of another Peptide Chain Release Factors domain linked to the protein family; furthermore, this domain plays an important role in newly synthesized polypeptide chains released from peptidyl-tRNA [63]. BdLHP1 contains another ER membrane protein $\mathrm{SH} 3$, which is associated with membrane localized chaperones. PpLHP1 comprises an additional ostepontin domain. 


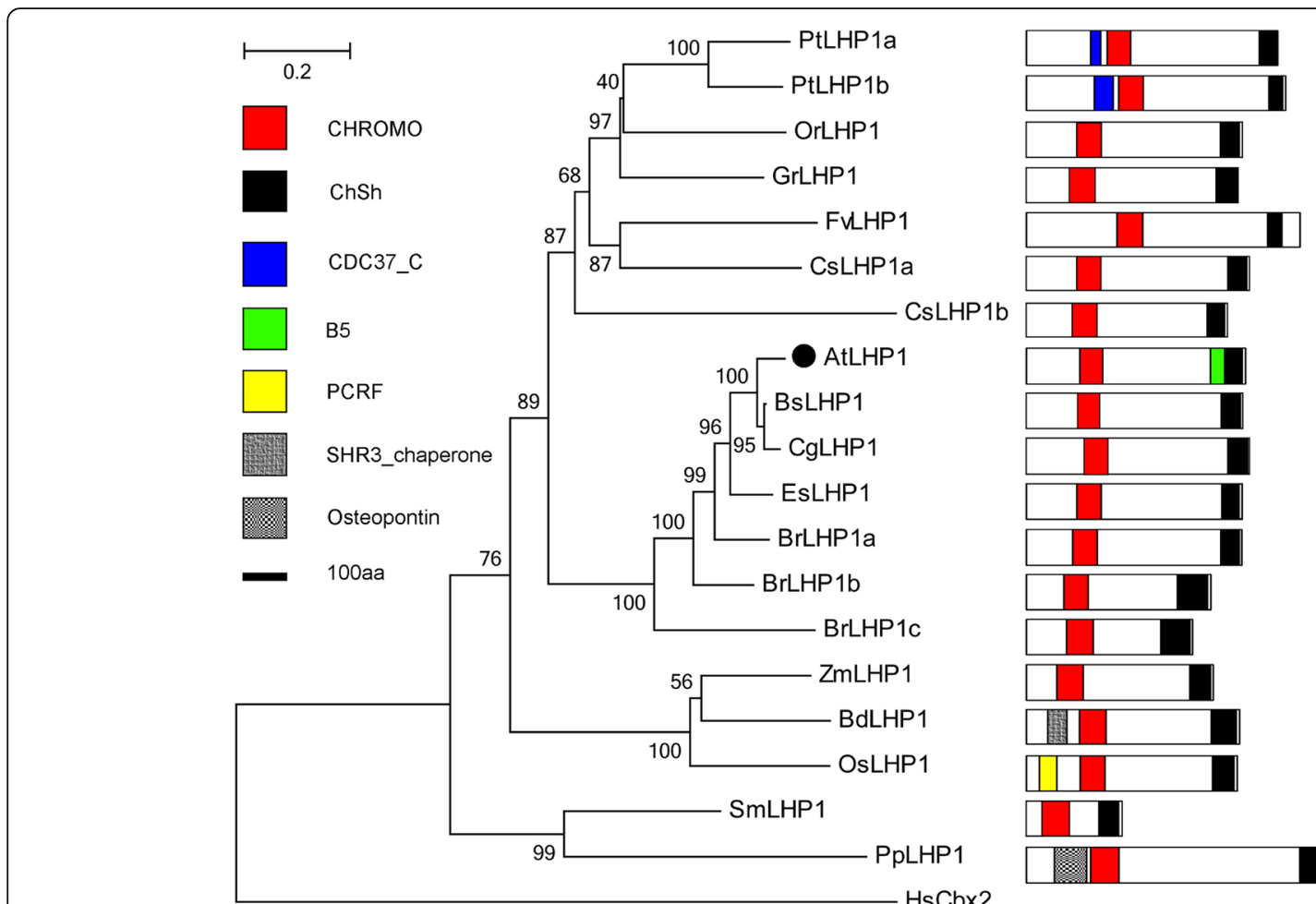

Fig. 4 Phylogenetic tree of LHP1 proteins in the Green Lineage. Plant LHP1 homologs exist in higher plant, and not in alga. CHROMO and CHSH domains are the essential features of LHP1 proteins

\section{PRC1 associated factor: EMF1}

EMF1 and VRN1 are specifically found in dicotyledon species [27, 40]. Both EMF1 and VRN1 are non-sequence specific DNA-binding proteins that regulate gene expression during flower organ development. Aubert et al. regarded EMF1 as a novel protein involved in the controlling of shoot architecture and flowering in Arabidopsis; furthermore, EMF1 loss-of-function mutants cause accelerated embryonic to reproductive development transition [64]. EMF1 and EMF2 participate in the PcG-mediated silencing of flower homeotic genes and are crucial for vegetative development $[32,65]$. EMF1, ATX1, and ULT1 can work together to maintain chromatin integrity and prevent precocious seed gene expression after germination [66]. EMF1 is associated with an $\mathrm{H} 3 \mathrm{~K} 27 \mathrm{me} 3$ reader that is required for H3K27me3 [23, 67]. EMF1, LHP1, and histone $\mathrm{H} 3$ lysine-4 demethylase can form an EMF1c complex to play important roles in the regulation of MIR172 and Flowering Locus $T$ (FT) $[28,68]$.

Each species harbors a single EMF1 homologous gene except for cucumber, cotton and Eutrema with two, and cabbage with four. Phylogenetic analysis shows that EMF1 are well conserved in dicotyledon, but may lack representative or intact domains in the Pfam and SMART database. Protein sequence alignment suggests that six conserved motifs, especially motif 4, 5, and 6 (Fig. 5, Additional file 4), and functions of which are unknown.
PRC1 associated factor: VRN1

VRN1 and VAL1/2/3 are plant-specific components of PRC1, and are subclades of the plant specific B3 domain transcription factor family (Additionalfile 5). Similar to EMF1, Arabidopsis VRN genes can mediate vernalization and play a major role in vegetative to reproductive phase transition in response to prolonged cold treatment. VRN1 localizes in the nucleus and is sequence-nonspecific in DNA binding, targeting at FLC, and FT2 [69-71]. Loss-offunction mutants show similar phenotypes to other PRC1 mutants [72].

VRN1 and its homologs are subgrouped into the two clades, AtVRN1a/RTV1 and AtVRN1b/1c/1d. The B3 domain is possibly a special domain of the VRN1 family (71, Fig. 6). AtVRN1, which is named AtVRN1a in this study, is characterized with two B3 domains, is found only in higher plant species and specifically binds to DNA [40]. In the current study, five VRN1 homologs are identified in Arabidopsis, and multiple homologs are also found in other dicotyledons by BlastP. The domain organization showed that AtVRN1a and its homologs consist of two B3 domains (Fig. 6). AtRTV1, AtVRN1b/ 1c/1d and their homologs mainly in group-II show a loss in the second B3 domain, which may be important for its functions [71]. This domain is replaced by the BfiI C_EcoRII_N_B3 super family, which contains an N-terminal DNA binding domain of type IIE restricted 


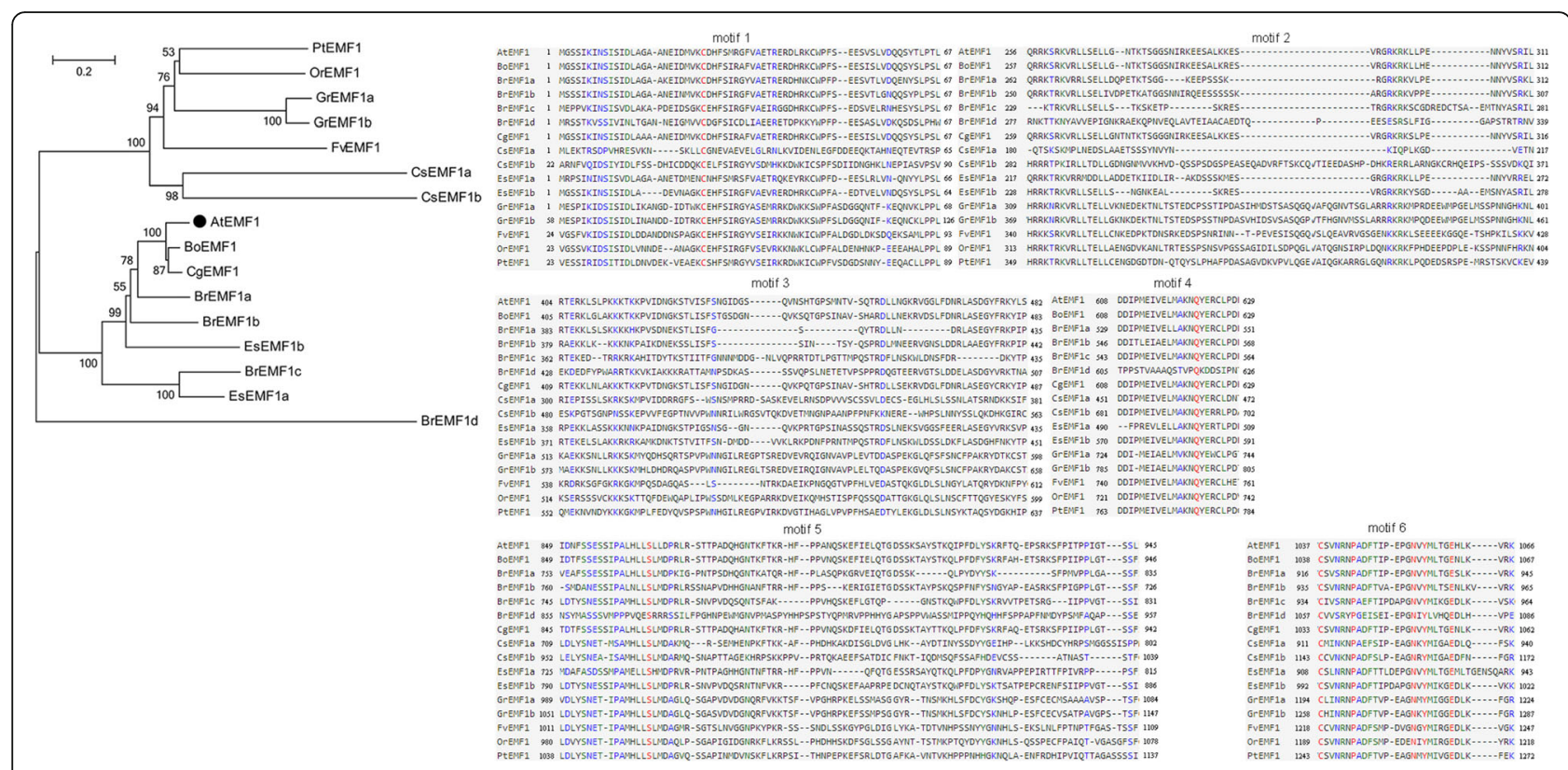

Fig. 5 Phylogenetic tree of EMF1 proteins in the Green Lineage. Plant EMF1 homologs only exist in dicotyledons. Six motifs are detected in plant EMF1 proteins

endonuclease EcoRII-like proteins, a C-terminal DNA binding domain of type IIS restricted endonuclease Bfillike proteins, and plant-specific B3 proteins [73].

\section{PRC1 associated factor: VAL1/2/3}

VAL proteins, which are identified as a transcriptional repressor, are required for global repression of embryonic genes expression [74]. The seedlings of val1 val2 double mutant can form embryo-like proliferations in roots and apical meristem, but not in leaves. Val2/val3 mutants exhibit similar dominant effects in the val1 homozygous mutant plants [74]. In val1 mutants, 39\% of transcripts in the FUSCA3 regulator are depressed, whereas the core LAFL network transcription factors are not. All putative targeted transcripts of VAL1 act through epigenetic and/ or transcriptional repression [75]. Additionally, VAL1 and VAL2 are involved in vernalization via PcG. VAL proteins work together with BMI1 to mediate the monoubiquitylation of H2AK119, and initiate the repression of seed maturation genes [17]. VAL proteins mediate repression through recruiting a histone deacetylase complex to LEC1/AFL genes [76]. VAL1 represses FLC transcription by promoting histone deacetylation [77]. VAL1 downregulates AGL15 by H3K27me3 deposition at the upstream sequences of $A G L 15$ [78].

Except for B3 and zf-CW domains, which are possible special domains for the VAL1/2/3 family, most VAL1/2/3 homologs carry additional Zinc finger motifs, such as PHD and ZnF-GATA, at the 3 '-terminal (Fig. 7). The VAL1-B3 domain is necessary to interact with the canonical Sph/RY element within AGL15 and FLC [78-80]. The
$\mathrm{zf}-\mathrm{CW}$ domain is a member of the histone modification reader modules for epigenetic regulation [81]. In the current study, the VRN1 family is just found in dicotyledons (Fig. 6) and its homologs contain one or two B3 domains. By contrast, VAL1/2/3 proteins are found from alga to angiosperm, and only one B3 domain. Furthermore, VAL1/2/3 proteins can be clustered into three groups (Fig. 7). Group-I-containing VAL1 homologs are only found in dicotyledons; Group-II-containing VAL2 homologs and Group-III-containing VAL3 homologs are found in both dicotyledons and monocotyledons. As indicated by our results Alga and fern show only one VAL homologous protein, whereas moss demonstrates five members, and O. lucimarinus exhibits none.

\section{PRC1 associated factor: AL1-7}

AL proteins, carrying a conserved PHD domain, were identified as a transcription factor [82]. Arabidopsis Alfin proteins are regarded as $\mathrm{H} 3 \mathrm{~K} 4 \mathrm{me} 2 / 3$ readers $[36,38]$ and function as novel partners of AtRING1 and AtBMI1 [36]. The AL protein is involved in many developmental processes, such as enhancement of MsPRP2 expression in alfalfa roots and contributes to salt tolerance [83]. In Arabidopsis, both AL1 and AL5 can bind to the promoter regions of target genes and suppress multiple negative factors to confer abiotic stress tolerance [84, 85]. Arabidopsis AL6 is implicated in regulating the expression of root hair elongation-related transcripts on phosphate starvation; furthermore, this process is due to its PHD domain that can bind to H3K4me3, which is an epigenetic regulation strategy for low phosphate availability [86, 87]. However, 


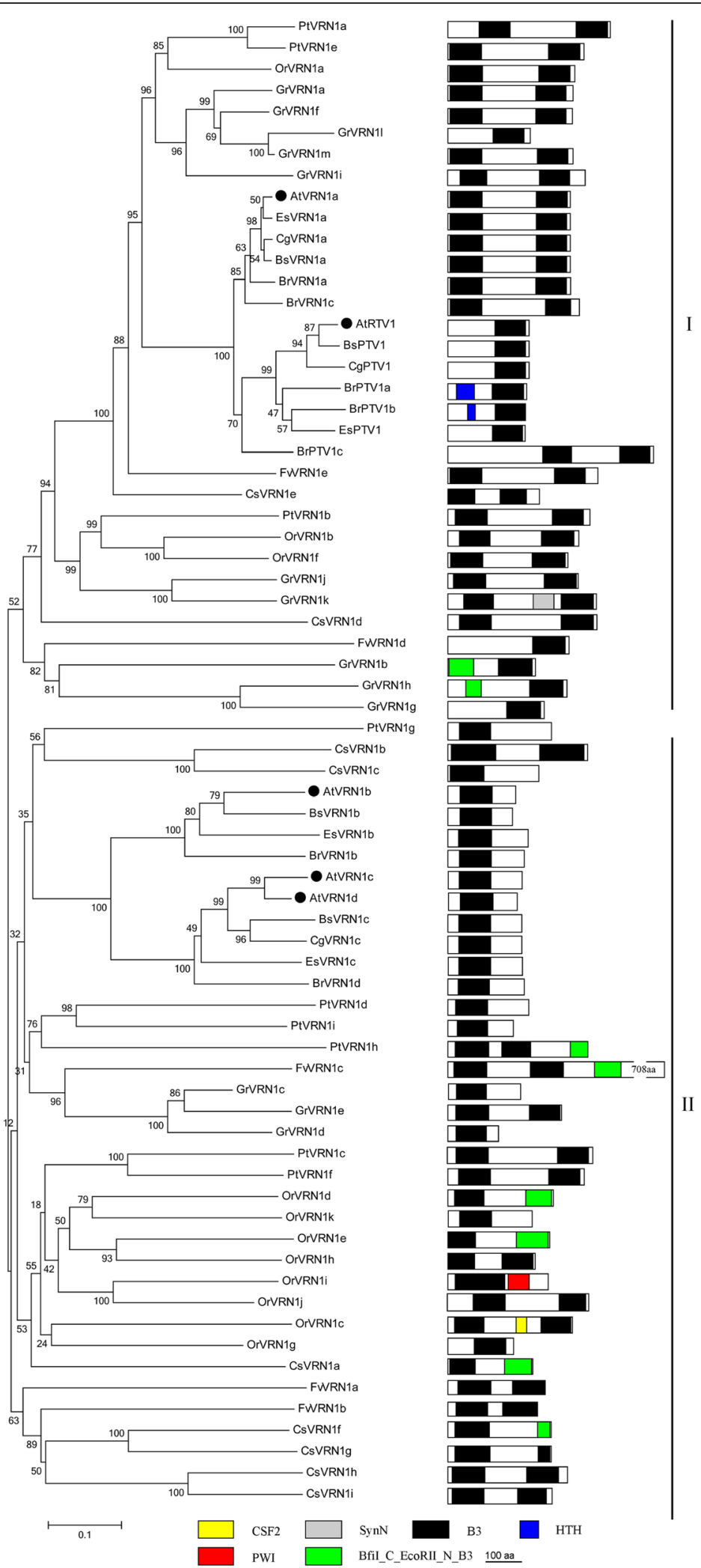

Fig. 6 Phylogenetic tree of VRN1 proteins in the Green Lineage. Plant VRN1 homologs only exist in dicotyledons and were subgrouped into the two clades: Group-I AtVRN1a/RTV1 and group-II AtVRN1b/1c/1d. B3 domain is the essential features of plant VRN1 proteins 


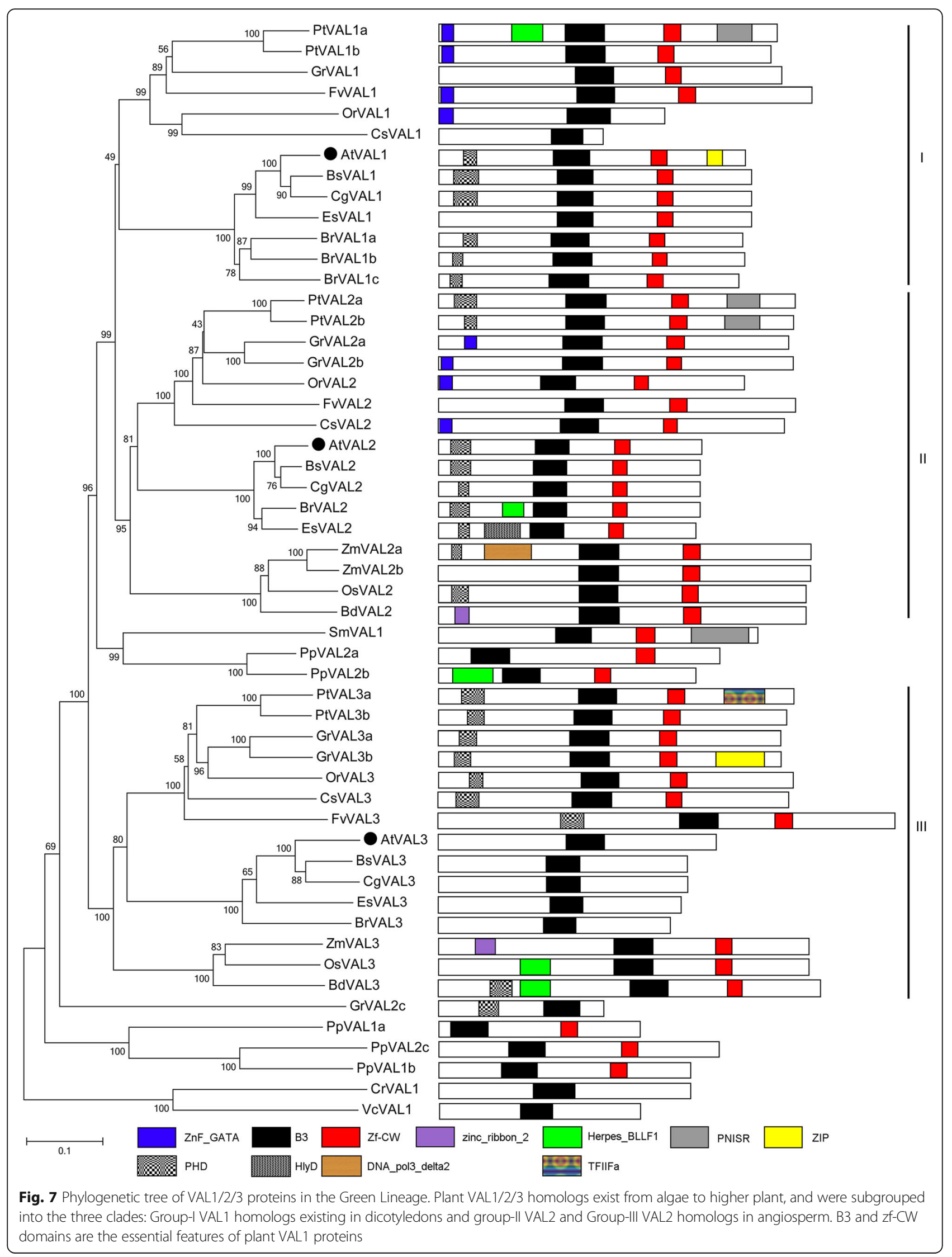


AtAL7 plays a negative role in salt tolerance [88]. In the current study, the AL and ING families share PHD domain, and the phylogenetic tree shows that they belong to different branches, suggesting their close relation (Additional file 6). The ALs family is the largest PRC1 associated factor family. Arabidopsis comprise seven ALs that can be divided into three groups, AtAL1/2, AtAL3/4/ 5 and AtAL6/7 [84, 88]. In the current study, the AL proteins of seed plants can be divided into three groups, namely, Group-I (AL1/2), Group-II (AL3/4/5), and Group-III (AL6/7). The AL proteins of spore plant are located on the bottom of the phylogenetic tree (Fig. 7). Maize and cotton comprise more AL protein members than others species.

Aside from FvAL5, which comprise 687aa with three Alfin domains and one PHD domain, most plants are extremely conserved in domain organizations, that is one Alfin domain and PHD, and exhibit a sequence length of approximately 230-300aa. (Fig. 7, Additional file 1). One Alfin and PHD domians, the special domain for the AL family, are distributed on the $\mathrm{N}$ - or $\mathrm{C}$ - terminus of the proteins. The PAL motif, located in the Alfin domain, of AL2 and AL7 proteins can bind to RING1 and BMI1 [35]. PHD-finger proteins are universally found in eukaryotes and act as key players in regulating transcription and chromatin structure $[38,89]$. PHD finger is required for H3K4me3/2 binding in the AL and ING families [38].

\section{PRC1 associated factor: ING1/2}

AL proteins exist only in plants whereas ING proteins are widely distributed in yeast, animals and plants. ING was first identified in mammals, and all five ING proteins can bind to H3K4me3/2 through PHD fingers and act as components of histone modifications [38, 90]. However, these proteins have rarely studied in plants. Similar to AL proteins, conserved AtING proteins can recognize H3K4me3/2 mediated by PHD fingers, whereas the biological functions of AtING are unknown [38].

Most plants contain two ING genes (Fig. 8). ING proteins carry an N-terminal ING domain that binds to unmodified $\mathrm{H} 3$ tails, and a C-terminal PHD domain that is necessary for binding $\mathrm{H} 3 \mathrm{~K} 4 \mathrm{me} 2 / 3$ [38]. In contrast with previous works, we identified VcING1/2, OlING1 and CrING1/2 homologs in green algae. The PHD fingers in VcING2 and CrING2 are replaced by the Tudor domains, which are also implicated in protein-protein interactions. The Tudor domain may bind to the symmetrically dimethylated arginines of arginine-glycine rich sequences and histone H4 dimethylated at Lys20 [91].

\section{PRC1 associated factor: EBS/SHL}

EBS and SHL are BAH-domain-containing proteins [23], which are only found in plant kingdom and widely distributed from lower to higher plants (Fig. 1, [92]).
Arabidopsis EBS proteins are negative transcriptional regulators, and $e b s$ mutations result in early flowering phenotypes [93]. EBS and SHL bind to different floral integrators, EBS regulating FT and SHL repressing SOC1 $[94,95]$. EBS and SHL act redundantly in regulation of seed dormancy [96]. EBS/SHL are H3K27me3 readers that can also bind H3K4me3 [31, 97].

EBS/SHL proteins, sub-grouped to two clades (group-I EBS homologs and group-II SHL homologs). Group-I exists in higher plants, but group-II only in angiosperm. Three EBS homologs, PpEBSe/d/f from moss and EBS/ SHL homologs from algae are at the bottom of the phylogenetic tree. Most species comprise a single SHL copy, but multiple EBS copies, as reported in poplar, cotton and moss (Figs. 1 and 9). EBS/SHL proteins are highly conserved in length, which ranges from 199 to 336 aa (most are around $220 \mathrm{aa}$ ), and in domain organization, an $\mathrm{N}$-terminal BAH domain and a C-terminal PHD domain (Fig. 9). PHD finger is related to $\mathrm{H} 3 \mathrm{~K} 4 \mathrm{me} 2 / \mathrm{me} 3$, and $\mathrm{BAH}$ domain reads $\mathrm{H} 3 \mathrm{~K} 27 \mathrm{me} 2 / \mathrm{me} 3$ mark. In general, H3K4me3 correlates with transcriptional activation, whereas H3K27me3 correlates with gene silencing in plants and animals. EBS possesses a BAH domain and a PHD domain that reads and affects H3K27me2/me3 and H3K4me/me3 marks, respectively [31, 94]. Furthermore, $\mathrm{BAH}$ domain, not the PHD finger, mediates the interaction of SHL or EBS with EMF1 [23]. BAH-H3K27me3 and PHD-H3K4me3 interactions are important for SHLmediated floral repression [97]. EBS/SHL balances active and repressive chromatin states.

\section{Discussion}

The presence of PRC2 complex in lower plants including algae has been reported [41, 98]. PRC1 is also evolutionarily ancient, and its subunits are found in mammals, plants, insects, and other species, but are lost in several primitive organisms [41]. Sanchez-Pulido et al. proposed that RING1 has originated before the last eukaryotic common ancestor, and BMI1 has duplicated and diverged from RING1 proteins [48]. In plants, RING1 and BMI1 are present in chlorophyte, moss, monilophyte, lycophyte, gymnosperm and angiosperm genomes. However, LHP1 is found only in land plants, but not in chlorophyte. EMF1 and VRN1 have originated from the ancestor of seed plants [40]. Chen et al. inferred that RING1 and BMI1 have originated in mosses [42].

In our dataset, the core components RING1 and BMI1 homologs are detected in algae, except in the most compact eukaryotic O. lucimarinus. LHP1 is only found in land plants, but not in algae. These results are in agreement with Berke and Snel [40] (Fig. 1). Consistent with the results of Molitor and Shen [27], but contrary to those of Berke and Snel [40], the associated components VRN1 were exclusively found in eudicots, but not in all 


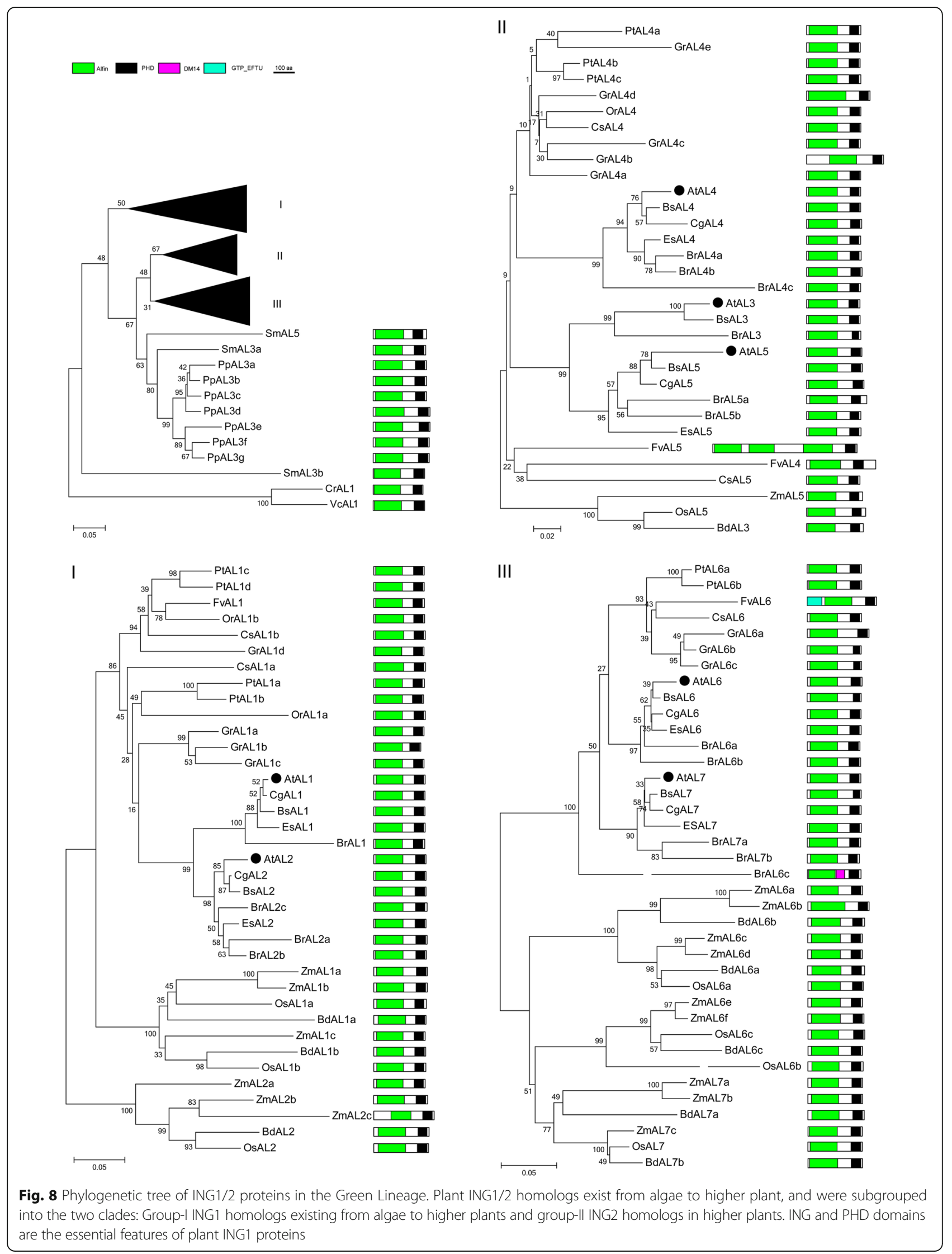




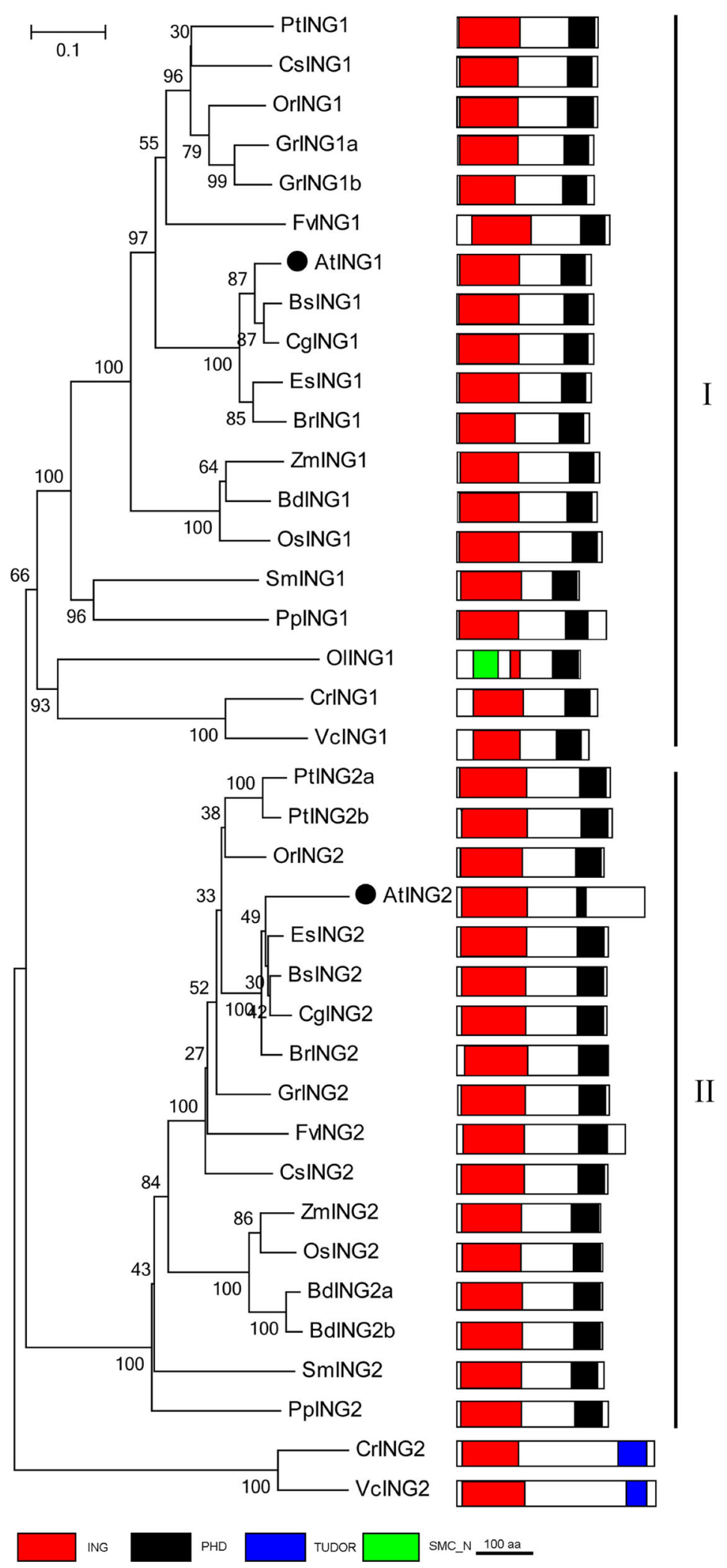

Fig. 9 Phylogenetic tree of EBS/SHL proteins in the Green Lineage. Plant EBS/SHL homologs exist from algae to higher plant, and were subgrouped into the two clades: Group-I EBS homologs existing in higher plants and group-II SHL homologs in angiosperm. BAH and PHD domains are the essential features of plant EBS/SHL proteins 
seed plants (Fig. 1); Maize VRN1, a MADS-box transcription factor, is homologous to Arabidopsis APETALA 1 [99, 100] and not to AtVRN1. Ubiquitous AL proteins are regarded as PRC1 associated factors [36], and ING1/2 exhibits similar functions, thus both these proteins are regarded as PRC1 associated components in this paper (Fig. 1). AL1-7, ING1/2, and $\mathrm{EBS} / \mathrm{SHL}$ are found in all plant species, from algae to higher plants (Fig. 1, Additional file 1). AL proteins can interact with AtRING1 and AtBMI1 and form AL-PHD-PRC1 complexes to repress the propagation ofH3K4me3. Berke and Snel suggested that the absence of PRC1 subunits is not detrimental for plants, notwithstanding their important roles in other biological processes, such as in LHP1 [40].

Parihar et al. suggested that LHP1 exists in mosses and is conserved in land plants [101]. The sporadic occurrence of subunits in chlorophytes and mosses strongly suggests that the proteins, LHP1, RING1 and BMI1, may demonstrate primitive functional roles in moss [40]. LHP1, as a reader of H3K27me3, may play a role as the fly Pc in a PRC1-like complex [36]. We anticipated that the RING1/BMI1-PRC1 complex is sufficient for alga H2Aub1, but LHP1 is not necessary for alga because of its absence in alga (Fig. 1). In land plants, LHP1 is involved in multiple basic developmental processes including organogenesis and floral transition, which has not been reported in alga. AL6/7 are H3K4me3 readers and PHD domain-containing $\mathrm{AL}$ proteins can recruit AtBMI1b and AtRING1ato mediate the transition from H3K4me3 to H3K27me3 [38, 102]. Furthermore, EBS/ SHL functions as the H3K4me3 or H3K27me3 reader $[23,31]$. It was suggested that ALs or EBS/SHL may make up for the absence of LHP1 in algae. ING induces targeted histone acetylation where the PRC1 complex is not involved [103, 104]. Both AL and ING exist in algae because both gene families may bind to target genes, except PRC1. Furthermore, AL and ING are involved in special functions, experimental evidence for which is however needed.

Interestingly, VAL/AL/ING and RING1/BMI1 co-exist from lower to higher plants (Figs. 1, 7, 8, 10). RING1 and BMI1 are conserved in plants [27]. The number of RING and BMI1 homologous genes is relatively stable, but ALs and EBS/SHL exist greatly in higher plants, especially in polyploids (Fig. 1). VRN, VAL, AL, and ING proteins carrying B3 or PHD domain mainly function in binding histones or genes and are involved in epigenetic regulation related to PRC1 core components. EBS/SHL is a reader of $\mathrm{H} 3 \mathrm{~K} 4 \mathrm{me} 3$ or $\mathrm{H} 3 \mathrm{~K} 27 \mathrm{me} 3$. However, the associated factors may or may not be involved in the evolution of core PRC1.

PRC1 proteins are highly conserved in plant domain organization (Figs. 2, 3, 4, 5, 6, 7 and 10). Each subunit exhibits the following special domain organizations: RING1 and BMI1 share RING and RAWUL domains, LHP1 with CHROMO and ChSh domains, VRN1 with one or two B3 domain(s), VAL1/2/3 with B3 and zf-CW domains, AL1-7 with PHD and Alfin domain, and ING1/2 with PHD and ING domain, EBS/SHL with PHD and BAH domain (Additional files 7 and 8). Moreover, six new motifs are discovered in EMF1, and motifs 4, 5 and 6 are well conserved (Fig. 5, Additional file 4).

Gene duplication is one of the primary driving forces in the evolution of genomes and genetic systems, and is a major mechanism for the establishment of new gene functions and the generation of evolutionary novelty. Nonfunctionalization, neofunctionalization and subfunctionalization are the three alternative outcomes of duplicated genes [105]. RING1 has undergone two duplications in the monocot ancestor and then has subsequently undergone several species-specific duplications or losses. However, BMI1 has undergone three duplications in monocots and two duplications in eudicots [40]. In the current study, PRC1 components have multiple homologs in Arabidopsis, except for LHP1 and EMF1, which are conservations in numbers. Other PRC1 members often exhibit several homologs in monocot and dicot plants, except algae and Cruciferae. Brassica rapa, Gossypium rainondii, Populus trichocarpa, and Zea mays always harbor multiple members of BMI1, VRN1, VAL1/2/3, AL1-7, and EBS/SHL. BMI1a/1b is a result of a Brassicaceae-specific duplication, and BMI1c exists in Cruciferae, a member of the second eudicot orthologous group (Fig. 3) [40]. Polycomb proteins encoding genes are duplicated in plant evolution process. The most recent polyploidy event was specific to the Brassicaceae family [106].

B. rapa $(2 \mathrm{n}=38)$ has undergone whole genome triplication after divergence from Arabidopsis thaliana $(2 \mathrm{n}=10)$, and many PRC1 genes are duplicated $[107,108]$. For example, AtRING1a and AtRING1b exhibit conserved sequences and domain organizations. atring1a or atring $1 b$ mutants show normal phenotypes, but atring1;atring1b forms ectopic meristem, and AtRING1a and AtRING1b are of functional redundancy [45]. In the current study, $B$. rapa in Brassicaceae comprises three RING1 homologs, except for BrRING1b lacking the RAWUL domain (Fig. 2); three BMI1 homologs with well conserved domain organizations (Fig. 3); three LHP homologs with CHROMO and ChSh domains but lacking the B3 domain (Fig. 4). The associated factors EMF1, VAL1/2/3, AL1-7, and EBS/SHL family are composed of several members (Figs. 6 and 7). This phenomenon also occurs in cotton, poplar and maize. Several conserved members indicate possible functional redundancy, whereas the observed variation in domain organization suggests the genes may be functionally divergent. 


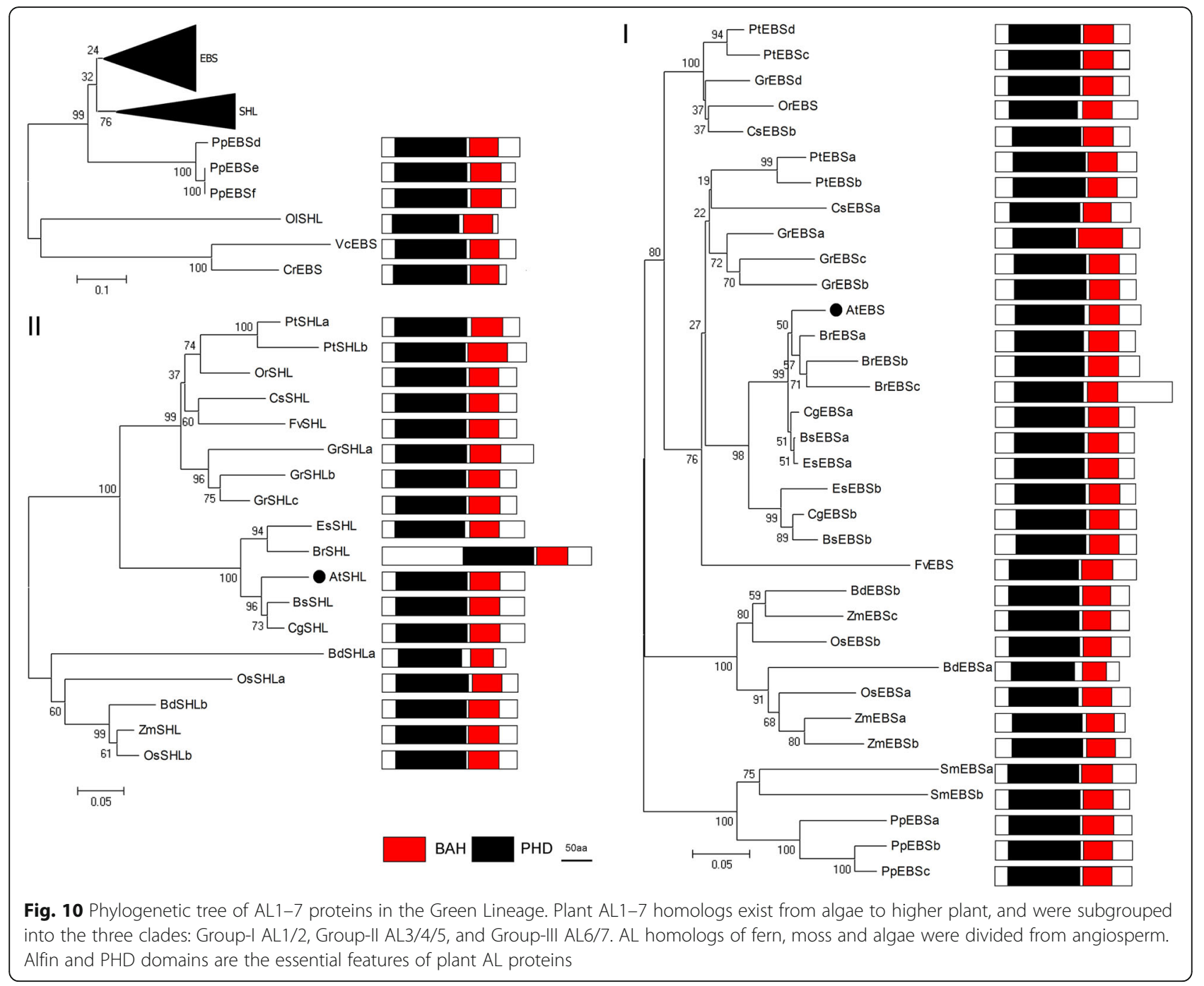

\section{Conclusions}

RING1, BMI1, VAL1/2/3, AL1-7, ING1/2, and EBS/SHL are found from algae to higher plants, However, LHP1 only exists in higher plants, and EMF1 and VRN1 are only found in eudicots. Most plants carry one homologous core component LHP1 and a single associated factor EMF1, but multiple members of RING1, BMI1, VRN1, VAL1/2/3, AL1-7, ING1/2 and EBS/SHL. Cabbage, cotton, poplar, orange, and maize often possess more gene copies than other species. The domain organizations are well conserved, except for individual members of proteins with multi-copies. Six motifs are also uncovered in EMF1. The results will facilitate functional studies on these important epigenetic regulatory genes in plants.

\section{Methods}

\section{PRC1 subunit identification}

The sequences of PRC1 proteins from A. thaliana were retrieved from the NCBI. The sequences then were used as queries to search other organisms (http://www.phytozome.net, version 12.0) by the BLASTp tools (http:// blast.ncbi.nlm.nih.gov). The sequences were then used as query to Blastp in TAIR (http://www.arabidopsis.org/ Blast/index.jsp), and sequences hitting to relevant PcG proteins of Arabidopsis were analyzed for their domain organizations (Additional file 1). The sequences were named based on their relationship to Arabidopsis homologous genes if they could be confirmed, or based on the location on the chromosome if the relationship could not be clearly determined.

Protein domain organization and phylogenetic analysis The protein sequences were collected to analyze domain organization by using NCBI-CD searches (http://ncbi.nlm. nih.gov/Structure/cdd/wrpsb.cgi) in the Pfam and SMART database. The low-complexity filter was turned off, and the Expect Value was set at 10 to detect short domains or 
regions of less conserved in this analysis. Sequences with special domain organizations were used to construct phylogenetic trees.

Multiple sequence alignments were performed using the ClustalW 2.0 program [109]. The resulting file was subjected to phylogenic analysis using the MEGA7.0 program [110]. The trees construction setting was dependent on full length protein sequences. Phylogenetic tree analysis was set as follows: neighbor joining as tree inference; sites as pairwise deletion included; substitution model consisting of P-distance (for BMI1, VRN1, VAL, AL and ING) or passion (for RING1,LHP1, EMF1, and EBS/SHL); and Bootstrap test of 1000 replicates for internal branch reliability.

\section{Additional files}

Additional file 1: Detailed information of PRC1 components in the green lineage. (XLSX $274 \mathrm{~kb}$ )

Additional file 2: Phylogenetic tree of BMI1 and RING1 domain containing proteins in the green lineage. (JPG $2829 \mathrm{~kb}$ )

Additional file 3: Sequence alignment of BMI1 and RING1 RAWUL domain in the green lineage. (JPG $2023 \mathrm{~kb}$ )

Additional file 4: Sequence alignment of EMF1 proteins in the green lineage. (JPG $5338 \mathrm{~kb}$ )

Additional file 5: Phylogenetic tree of VAL1 proteins in the green lineage. (JPG $2607 \mathrm{~kb}$ )

Additional file 6: Phylogenetic tree of AL1 and ING1 proteins in the green lineage. (JPG $3065 \mathrm{~kb}$ )

Additional file 7: Mark domains of PRC1 components. (XLSX $9 \mathrm{~kb}$ )

Additional file 8: Proteins sequence of PRC1 components. (DOCX $151 \mathrm{~kb}$ )

\section{Abbreviations}

AL1-7: Alfin-like 1-7; B3 domain: B3 DNA binding domain; BMI1: B lymphoma Mo-MLV insertion region 1 homolog; CHROMO: Chromatin Organization Modifier; ChSh: Chromo Shadow; EMF1: Embryonic Flower 1; H2Aub: Monoubiquitination; ING1/2: Inhibitor of growth; LHP1: Like heterochromatin Ppotein 1: Pc: Polycomb; PcG: Polycomb group: Ph: Polyhomeotic; PHD: Plant HomeoDomain; PRC1: Polycomb Repressive Complex 1; PRC1: Polycomb Repressive Complex 2; Psc: Posterior sex combs; RAWUL: Ring-finger And WD40 associated Ubiquitin-Like; RING1: Ring finger protein 1; Sce: Sex combs extra; VAL1/2/3: VP1/ABI3-Like 1/2/3;

VRN1: Vernalization 1

\section{Acknowledgments}

Not applicable.

\section{Authors' contributions}

$\mathrm{YH}$ contributed to the analysis of PRC1 core components and draft; $L, B-Y L$, C-FT and D-HC contributed to data capture and analysis of PRC1 associate components; W-HS and YR conceived and directed the study and wrote the final version of the manuscript; All authors read and approved the final manuscript

\section{Funding}

This work was funded by The National Key Research and Development Program of China (2016YFD0100202-8), Natural Science Foundation of Hunan Province (2019JJ40116), Scientific Research Project of Hunan Provincial Education Department (18 K041), and Training Program for Youth Backbone Teacher in University of Hunan Province (Resistance and Epigenetic Mechanism of Rice and Brassica 2015). The funding sources had no influence on the design of the study and collection, analysis, and interpretation of data and in writing the manuscript.

\section{Availability of data and materials}

The datasets used and/or analysed during the current study available from the corresponding author on reasonable request.

Ethics approval and consent to participate

The field trail experiments in the current study were permitted by the local government in China.

\section{Consent for publication}

Not applicable.

\section{Competing interests}

The authors declare that they have no competing interests.

\section{Author details}

${ }^{1}$ Key Laboratory of Crop Epigenetic Regulation and Development in Hunan Province, Hunan Agricultural University, Changsha 410128, China. ${ }^{2}$ International Associated Laboratory of CNRS-FU-HAU on Plant Epigenome Research, Hunan Agricultural University, Changsha 410128, China. ${ }^{3}$ Key Laboratory of Plant Genetics and Molecular Biology of Education Department of Hunan Province, Hunan Agricultural University, Changsha 410128, China. ${ }^{4}$ State Key Laboratory of Subtropical Silviculture, SFGA Engineering Research Center for Dendrobium catenatum (D. officinale), Zhejiang A\&F University, Hangzhou 311300, China. ${ }^{5}$ Institut de Biologie Mole'culaire des Plantes du CNRS, Universite' de Strasbourg, 12 rue du Ge'ne'ralZimmer, 67084

Strasbourg Cedex, France.

Received: 22 October 2018 Accepted: 13 June 2019

Published online: 28 June 2019

\section{References}

1. Pirrotta V. Polycombing the genome: $P c G$, trxG, and chromatin silencing. Cell. 1998:93(3):333-6.

2. Bemer M, Grossniklaus U. Dynamic regulation of Polycomb group activity during plant development. Curr Opin Plant Biol. 2012;15(5):523-9.

3. Grossniklaus U, Paro R. Transcriptional silencing by polycomb-group proteins. Cold Spring Harb Perspect Biol. 2014;6(11):a019331.

4. Zhao W, Huang Y, Zhang J, Liu M, Ji H, Wang C, et al. Polycomb group RING finger proteins $3 / 5$ activate transcription via an interaction with the pluripotency factor Tex10 in embryonic stem cells. J Biol Chem. 2017; 292(52):21527-37.

5. Maezawa S, Hasegawa K, Yukawa M, Sakashita A, Alavattam KG, Andreassen $\mathrm{PR}$, et al. Polycomb directs timely activation of germline genes in spermatogenesis. Genes Dev. 2017;31(16):1693-703.

6. Sheldon CC, Conn AB, Dennis ES, Peacock WJ. Different regulatory regions are required for the Vernalization-induced repression of FLOWERING LOCUS C and for the epigenetic maintenance of repression. Plant Cell. 2002;14(10): 2527-37.

7. Köhler C, Hennig L, Bouveret R, Gheyselinck J, Grossniklaus U, Gruissem W. Arabidopsis MSI1 is a component of the MEA/FIE Polycomb group complex and required for seed development. EMBO J. 2003;22(18):4804-14.

8. Ojolo SP, Cao S, Priyadarshani SVGN, Li W, Yan M, Aslam M, et al. Regulation of plant growth and development: a review from a chromatin remodeling perspective. Front Plant Sci. 2018;9:1232.

9. He Y, Li Z. Epigenetic environmental memories in plants: establishment, maintenance, and reprogramming. Trends Genet. 2018;34(11):856-66.

10. Exner V, Aichinger E, Shu H, Wildhaber T, Alfarano P, Caflisch A, et al. The chromodomain of LIKE HETEROCHROMATIN PROTEIN 1 is essential for H3K27me3 binding and function during Arabidopsis development. PLoS One. 2009;4(4):e5335.

11. Müller J, Verrijzer P. Biochemical mechanisms of gene regulation by polycomb group protein complexes. Curr Opin Genet Dev. 2009;19(2):150-8.

12. Ketel CS, Andersen EF, Vargas ML, Suh J, Strome S, Simon JA. Subunit contributions to histone methyltransferase activities of fly and worm polycomb group complexes. Mol Cel Biol. 2005;25(16):6857-68.

13. Nekrasov M, Wild B, Müller J. Nucleosome binding and histone methyltransferase activity of Drosophila PRC2. EMBO Rep. 2005;6(4):348-53.

14. Jones $\mathrm{A}$, Wang $\mathrm{H}$. Polycomb repressive complex 2 in embryonic stem cells: an overview. Protein Cell. 2010;1(12):1056-62.

15. Bratkowski M, Yang $X$, Liu X. An evolutionarily conserved structural platform for PRC2 inhibition by a class of Ezh2 inhibitors. Sci Rep. 2018;8(1):9092. 
16. Nekrasov M, Klymenko T, Fraterman S, Papp B, Oktaba K, Köcher T, et al. Pcl$\mathrm{PRC} 2$ is needed to generate high levels of $\mathrm{H} 3-\mathrm{K} 27$ trimethylation at Polycomb target genes. EMBO J. 2007;26(18):4078-88.

17. Yang C, Bratzel F, Hohmann N, Koch M, Turck F, Calonje M. VAL- and AtBMI1-mediated H2Aub initiate the switch from embryonic to postgerminative growth in Arabidopsis. Curr Biol. 2013;23:1324-9.

18. Wang $H$, Wang L, Erdjument-Bromage $H$, Vidal $M$, Tempst $P$, Jones RS, et al. Role of histone $\mathrm{H} 2 \mathrm{~A}$ ubiquitination in Polycomb silencing. Nature. 2004; 431(7010):873-8

19. Pherson M, Misulovin Z, Gause M, Mihindukulasuriya K, Swain A, Dorsett D. Polycomb repressive complex 1 modifies transcription of active genes. Sci Adv. 2017;3(8):e1700944.

20. Lehmann L, Ferrari R, Vashisht AA, Wohlschlegel JA, Kurdistani SK, Carey M. Polycomb repressive complex 1 (PRC1) disassembles RNA polymerase ॥ preinitiation complexes. J Biol Chem. 2012;287(43):35784-94.

21. Deshaies RJ, Joazeiro CA. RING domain E3 ubiquitin ligases. Annu Rev Biochem. 2009;78:399-434

22. Cao R, Tsukada Y, Zhang Y. Role of Bmi-1 and Ring1A in H2A ubiquitylation and Hox gene silencing. Mol Cell. 2005;20(6):845-54

23. Li Z, Fu X, Wang Y, Liu R, He Y. Polycomb-mediated gene silencing by the BAH-EMF1 complex in plants. Nat Genet. 2018;50(9):1254-61.

24. Arora M, Packard CZ, Banerjee T, Parvin JD. RING1A and BMI1 bookmark active genes via ubiquitination of chromatin-associated proteins. Nucleic Acids Res. 2016:44(5):2136-44.

25. Paschos K, Bazot Q, Lees J, Farrell PJ, Allday MJ. Requirement for PRC1 subunit BMI1 in host gene activation by Epstein-Barr virus protein EBNA3C. Nucleic Acids Res. 2019. https://doi.org/10.1093/nar/gky1323.

26. Völkel P, Le Faou P, Vandamme J, Pira D, Angrand PO. A human Polycomb isoform lacking the $\mathrm{pc}$ box does not participate to PRC1 complexes but forms protein assemblies and represses transcription. Epigenetics. 2012;7(5):482-91.

27. Molitor A, Shen WH. The polycomb complex PRC1: composition and function in plants. J Genet Genomics. 2013;40(5):231-8

28. Picó S, Ortiz-Marchena Ml, Merini W, Calonje M. Deciphering the role of POLYCOMB REPRESSIVE COMPLEX1 variants in regulating the acquisition of flowering competence in Arabidopsis. Plant Physiol. 2015;168(4):1286-97.

29. Yang X, Tong A, Yan B, Wang X. Governing the silencing state of chromatin: the roles of Polycomb repressive complex 1 in Arabidopsis. Plant Cell Physiol. 2017;58(2):198-206

30. Pasini D, Bracken AP, Jensen MR, Lazzerini E, Denchi EL, Helin K. Suz12 is essential for mouse development and for EZH2 histone methyltransferase activity. EMBO J. 2004;23(20):4061-71.

31. Yang Z, Qian S, Scheid RN, Lu L, Chen X, Liu R, et al. EBS is a bivalent histone reader that regulates floral phase transition in Arabidopsis. Nat Genet. 2018;50(9):1247-53.

32. Kim SY, Lee J, Eshed-Williams L, Zilberman D, Sung ZR. EMF1 and PRC2 cooperate to repress key regulators of Arabidopsis development. PLoS Genet. 2012;8(3):e1002512.

33. Wang Q, Shen WH. Chromatin modulation and gene regulation in plants: insight about PRC1 function. Biochem Soc Trans. 2018;46(4):957-66.

34. Lee K, Seo PJ. Dynamic epigenetic changes during plant regeneration. Trends Plant Sci. 2018;23(3):235-47.

35. Peng L, Wang L, Zhang Y, Dong A, Shen WH, Huang Y. Structural analysis of the Arabidopsis AL2-PAL and PRC1 complex provides mechanistic insight into active-to-repressive chromatin state switch. J Mol Biol. 2018;430(21):4245-59.

36. Molitor A, Bu Z, Yu Y, Shen WH. Arabidopsis AL PHD-PRC1 complexes promote seed germination through H3K4me3-to-H3K27me3 chromatin state switch in repression of seed developmental genes. PLoS Genet. 2014;10(1):e1004091.

37. Guérillon C, Larrieu D, Pedeux R. ING1 and ING2: multifaceted tumor suppressor genes. Cell Mol Life Sci. 2013;70(20):3753-72.

38. Lee WY, Lee D, Chung WI, Kwon CS. Arabidopsis ING and Alfin1-like protein families localize to the nucleus and bind to H3K4me3/2 via plant homeodomain fingers. Plant J. 2009;58(3):511-24.

39. Leighton $L$, Zhao Q, Li X, Dai C, Marshall PR, Liu S, et al. A functional role for the epigenetic regulator ING1 in activity-induced gene expression in primary cortical neurons. Neuroscience. 2018;369:248-60.

40. Berke L, Snel B. The plant Polycomb repressive complex 1 (PRC1) existed in the ancestor of seed plants and has a complex duplication history. BMC Evol Biol. 2015;15:44.

41. Schubert D. Evolution of Polycomb-group function in the green lineage. F1000Research. 2019;8(F1000 Faculty Rev):268.
42. Chen DH, Huang Y, Ruan Y, Shen WH. The evolutionary landscape of PRC core components in green lineage. Planta. 2016;243(4):825-46.

43. Alchanati I, Teicher C, Cohen G, Shemesh V, Barr HM, Nakache P, et al. The E3 ubiquitin-ligase Bmi1/Ring1A controls the proteasomal degradation of Top2a cleavage complex-a potentially sew drug target. PLoS One. 2009; 4(12):e8104.

44. Buchwald G, van der Stoop P, Weichenrieder O, Perrakis A, van Lohuizen M, Sixma TK. Structure and E3-ligase activity of the ring-ring complex of polycomb proteins Bmi1 and Ring1b. EMBO J. 2006;25(11): 2465-74.

45. Xu L, Shen WH. Polycomb silencing of KNOX genes confines shoot stem cell niches in Arabidopsis. Curr Biol. 2008;18(24):1966-71.

46. Chen D, Molitor A, Liu C, Shen WH. The Arabidopsis PRC1-like ring-finger proteins are necessary for repression of embryonic traits during vegetative growth. Cell Res. 2010;20(12):1332-44.

47. Li J, Wang Z, Hu Y, Cao Y, Ma L. Polycomb group proteins RING1A and RING1B regulate the vegetative phase transition in Arabidopsis. Front Plant Sci. 2017:8:867.

48. Sanchez-Pulido L, Devos D, Sung ZR, Calonje M. RAWUL: a new ubiquitinlike domain in PRC1 ring finger proteins that unveils putative plant and worm PRC1 orthologs. BMC Genomics. 2008;9:308.

49. Bratzel F, Yang C, Angelova A, López-Torrejón G, Koch M, del Pozo JC, et al. Regulation of the new Arabidopsis imprinted gene AtBMI1C requires the interplay of different epigenetic mechanisms. Mol Plant. 2012;5(1):260-9.

50. Bratzel F, López-Torrejón G, Koch M, Del Pozo JC, Calonje M. Keeping cell identity in Arabidopsis requires PRC1 RING-finger homologs that catalyze H2A monoubiquitination. Curr Biol. 2010;20(20):1853-9.

51. Qin F, Sakuma Y, Tran LS, Maruyama K, Kidokoro S, Fujita Y, et al. Arabidopsis DREB2A-interacting proteins function as RING E3 ligases and negatively regulate plant drought stress-responsive gene expression. Plant Cell. 2008; 20(6):1693-707.

52. Hirano M, Hirano T. Opening closed arms: long-distance activation of SMC ATPase by hinge-DNA interactions. Mol Cell. 2006;21:175-86.

53. Sanchez-Pulido L, Devos D, Sung ZR, Calonje M. RAWUL: a new ubiquitinlike domain in PRC1 ring finger proteins that unveils putative plant and worm PRC1 orthologs. BMC Genomics. 2008;9:308.

54. Alatzas A, Foundouli A. Distribution of ubiquitinated histone H2A during plant cell differentiation in maize root and dedifferentiation in callus culture. Plant Sci. 2006;171:481-7.

55. Zhang Z, Li J, Tang Z, Sun X, Zhang H, Yu J, et al. Gnp4/LAX2, a RAWUL protein, interferes with the OsIAA3-OSARF25 interaction to regulate grain length via the auxin signaling pathway in rice. J Exp Bot. 2018;69(20):4723-37.

56. Wong SJ, Gearhart MD, Taylor AB, Nanyes DR, Ha DJ, Robinson AK, et al. KDM2B recruitment of the Polycomb group complex, PRC1.1, requires cooperation between PCGF1 and BCORL1. Structure. 2016;24(10):1795-801.

57. Feng J, Lu J. LHP1 could act as an activator and a repressor of transcription in plants. Front Plant Sci. 2017:8:2041.

58. Wiley EA, Horrell S, Yoshino A, Schornak CC, Bagnani C, Chalker DL. Diversification of HP1-like chromo domain proteins in Tetrahymena thermophila. J Eukaryot Microbiol. 2018;65(1):104-16.

59. Libault M, Tessadori F, Germann S, Snijder B, Fransz P, Gaudin V. The Arabidopsis LHP1 protein is a component of euchromatin. Planta. 2005;222(5):910-25.

60. Zemach A, Li Y, Ben-Meir H, Oliva M, Mosquna A, Kiss V, et al. Different domains control the localization and mobility of LIKE HETEROCHROMATIN PROTEIN1 in Arabidopsis nuclei. Plant Cell. 2006;18(1):133-45.

61. Dangwal M, Kapoor S, Kapoor M. The PpCMT chromomethylase affects cell growth and interacts with the homolog of LIKE HETEROCHROMATIN PROTEIN 1 in the moss Physcomitrella patens. Plant J. 2014;77(4):589-603.

62. Gaudin V, Libault M, Pouteau S, Juul T, Zhao G, Lefebvre D, et al. Mutations in LIKE HETEROCHROMATIN PROTEIN 1 affect flowering time and plant architecture in Arabidopsis. Development. 2001;128(23):4847-58.

63. Craigen WJ, Cook RG, Tate WP, Caskey CT. Bacterial peptide chain release factors: conserved primary structure and possible frameshift regulation of release factor 2. Proc Natl Acad Sci U S A. 1985;82(11):3616-20.

64. Aubert D, Chen L, Moon YH, Martin D, Castle LA, Yang CH, et al. EMF1, a novel protein involved in the control of shoot architecture and flowering in Arabidopsis. Plant Cell. 2001;13(8):1865-75.

65. Chen L, Cheng JC, Castle L, Sung ZR. EMF genes regulate Arabidopsis inflorescence development. Plant Cell. 1997;9(11):2011-24.

66. Xu F, Kuo T, Rosli Y, Liu MS, Wu L, Chen LO, et al. Trithorax group proteins act together with a Polycomb group protein to maintain chromatin 
integrity for epigenetic silencing during seed germination in Arabidopsis. Mol Plant. 2018;11(5):659-77.

67. Pu L, Liu MS, Kim SY, Chen LF, Fletcher JC, Sung ZR. EMBRYONIC FLOWER1 and ULTRAPETALA1 act antagonistically on Arabidopsis development and stress response. Plant Physiol. 2013;162(2):812-30.

68. Wang Y, Gu X, Yuan W, Schmitz RJ, He Y. Photoperiodic control of the floral transition through a distinct polycomb repressive complex. Dev Cell. 2014; 28(6):727-36.

69. Levy YY, Mesnage S, Mylne JS, Gendall AR, Dean C. Multiple roles of Arabidopsis VRN1 in vernalization and flowering time control. Science. 2002; 297(5579):243-6.

70. Liu H, Song S, Xing Y. Beyond heading time: FT-like genes and spike development in cereals. J Exp Bot. 2019;70(1):1-3.

71. King GJ, Chanson AH, McCallum EJ, Ohme-Takagi M, Byriel K, Hill JM, et al. The Arabidopsis B3 domain protein VERNALIZATION1 (VRN1) is involved in processes essential for development, with structural and mutational studies revealing its DNA-binding surface. J Biol Chem. 2013;288(5):3198-207.

72. Holec S, Berger F. Polycomb group complexes mediate developmental transitions in plants. Plant Physiol. 2012;158(1):35-43.

73. Golovenko D, Manakova E, Zakrys L, Zaremba M, Sasnauskas G, Gražulis S, et al. Structural insight into the specificity of the B3 DNA-binding domains provided by the co-crystal structure of the C-terminal fragment of Bfil restriction enzyme. Nucleic Acids Res. 2014;42(6):4113-22.

74. Suzuki M, Wang HHY, MCCarty DR. Repression of the LEAFY COTYLEDON 1/ B3 regulatory network in plant embryo development by VP1/ABSCISIC ACID INSENSITIVE 3-LIKE B3 genes1. Plant Physiol. 2007;143(2):902-11.

75. Schneider A, Aghamirzaie D, Elmarakeby H, Poudel AN, Koo AJ, Heath LS, et al. Potential targets of VIVIPAROUS1/ABI3-LIKE1 (VAL1) repression in developing Arabidopsis thaliana embryos. Plant J. 2016;85(2):305-19.

76. Jia H, Suzuki M, McCarty DR. Regulation of the seed to seedling developmental phase transition by the LAFL and VAL transcription factor networks. Wiley Interdiscip Rev Dev Biol. 2014;3(1):135-45.

77. Qüesta Jl, Song J, Geraldo N, An H, Dean C. Arabidopsis transcriptional repressor VAL1 triggers Polycomb silencing at FLC during vernalization. Science. 2016;353(6298):485-8.

78. Chen N, Veerappan V, Abdelmageed H, Kang M, Allen RD. HSI2NAL1 silences AGL15 to regulate the developmental transition from seed maturation to vegetative growth in Arabidopsis. Plant Cell. 2018:30(3):600-19.

79. Tsukagoshi H, Saijo T, Shibata D, Morikami A, Nakamura K. Analysis of a sugar response mutant of Arabidopsis identified a novel B3 domain protein that functions as an active transcriptional repressor. Plant Physiol. 2005; 138(2):675-85.

80. Sasnauskas G, Kauneckaite K, Siksnys V. Structural basis of DNA target recognition by the B3 domain of Arabidopsis epigenome reader VAL1. Nucleic Acids Res. 2018:46(8):4316-24.

81. He F, Umehara T, Saito K, Harada T, Watanabe S, Yabuki T, et al. Structural insight into the zinc finger $\mathrm{CW}$ domain as a histone modification reader. Structure. 2010;18(9):1127-39.

82. Qu $\sqcup$, Zhu YX. Transcription factor families in Arabidopsis: major progress and outstanding issues for future research. Curr Opin Plant Biol. 2006;9(5):544-9.

83. Bastola DR, Pethe W, Winicov I. Alfin 1, a novel zinc-finger protein in alfalfa roots that binds to promoter elements in the salt inducible MsPRP2 gene. Plant Mol Biol. 1998;38(6):1123-35.

84. Wei W, Zhang YQ, Tao JJ, Chen HW, Li QT, Zhang WK, et al. The Alfin-like homeodomain finger protein AL5 suppresses multiple negative factors to confer abiotic stress tolerance in Arabidopsis. Plant J. 2015;81(6):871-83.

85. Tao JJ, Wei W, Pan WJ, Lu L, Li QT, Ma JB, et al. An Alfin-like gene from Atriplex hortensis enhances salt and drought tolerance and abscisic acid response in transgenic Arabidopsis. Sci Rep. 2018;8(1):2707.

86. Chandrika NN, Sundaravelpandian K, Yu SM, Schmidt W. ALFIN-LIKE 6 is involved in root hair elongation during phosphate deficiency in Arabidopsis. New Phytol. 2013;198(3):709-20.

87. Chandrika NN, Sundaravelpandian K, Schmidt W. A PHD in histone language: on the role of histone methylation in plant responses to phosphate deficiency. Plant Signal Behav. 2013;8(6):e24381.

88. Song Y, Gao J, Yang F, Kua CS, Liu J, Cannon CH. Molecular evolutionary analysis of the Alfin-like protein family in Arabidopsis lyrata, Arabidopsis thaliana, and Thellungiella halophila. PLoS One. 2013;8(7):e66838.

89. Wang Q, Liu J, Wang Y, Zhao Y, Jiang H, Cheng B. Systematic analysis of the maize PHD-finger gene family reveals a subfamily involved in abiotic stress response. Int J Mol Sci. 2015;16(10):23517-44.
90. Garkavtsev I, Kazarov A, Gudkov A, Riabowol K. Suppression of the novel growth inhibitor p33|NG1 promotes neoplastic transformation. Nat Genet. 1996; 14(4):415-20

91. Lee J, Thompson JR, Botuyan MV, Mer G. Distinct binding modes specify the recognition of methylated histones H3K4 and H4K20 by JMJD2A-tudor. Nat Struct Mol Biol. 2008;15(1):109-11.

92. Mikulski P, Komarynets O, Fachinelli F, Weber APM, Schubert D. Characterization of the Polycomb-group mark H3K27me3 in unicellular algae. Front Plant Sci. 2017:8:607.

93. Gómez-Mena C, Piñeiro M, Franco-Zorrilla JM, Salinas J, Coupland G, Martínez-Zapater JM. Early bolting in short days: an Arabidopsis mutation that causes early flowering and partially suppresses the floral phenotype of leafy. Plant Cell. 2001;13(5):1011-24.

94. López-González L, Mouriz A, Narro-Diego L, Bustos R, Martínez-Zapater JM, Jarillo JA, et al. Chromatin-dependent repression of the Arabidopsis floral integrator genes involves plant specific PHD-containing proteins. Plant Cell. 2014;26(10):3922-38.

95. Pineiro M, Gomez-Mena C, Schaffer R, Martinez-Zapater JM, Coupland G. EARLY BOLTING IN SHORT DAYS is related to chromatin remodeling factors and regulates flowering in Arabidopsis by repressing FT. Plant Cell. 2003;15:1552-62.

96. Narro-Diego L, López-González L, Jarillo JA, Piñeiro M. The PHD-containing protein EARLY BOLTING IN SHORT DAYS regulates seed dormancy in Arabidopsis. Plant Cell Environ. 2017;40(10):2393-405.

97. Qian S, Lv X, Scheid RN, Lu L, Yang Z, et al. Dual recognition of H3K4me3 and H3K27me3 by a plant histone reader SHL. Nat Commun. 2018;9(1):2425.

98. Huang Y, Chen DH, Liu BY, Shen WH, Ruan Y. Conservation and diversification of polycomb repressive complex 2 (PRC2) proteins in the green lineage. Brief Funct Genomics. 2017;16(2):106-19.

99. Yan L, Loukoianov A, Tranquilli G, Helguera M, Fahima T, Dubcovsky J. Positional cloning of the wheat vernalization gene VRN1. Proc Natl Acad Sci U S A. 2003;100(10):6263-8.

100. Golovnina KA, Kondratenko EY, Blinov AG, Goncharov NP. Molecular characterization of vernalization loci VRN1 in wild and cultivated wheats. BMC Plant Biol. 2010;10:168.

101. Parihar V, Arya D, Walia A, Tyagi V, Dangwal M, Verma V, et al. Functional characterization of LIKE HETEROCHROMATIN PROTEIN 1 in the moss Physcomitrella patens: its conserved protein interactions in land plants. Plant J. 2019;97(2):221-39.

102. Bemer M. Unraveling the complex epigenetic mechanisms that regulate gene activity. Methods Mol Biol. 2018;1675:205-31.

103. Feng $X$, Hara Y, Riabowol K. Different HATS of the ING1 gene family. Trends Cell Biol. 2002;12(11):532-8.

104. Vieyra D, Loewith R, Scott M, Bonnefin P, Boisvert FM, Cheema P, et al. Human ING1 proteins differentially regulate histone acetylation. J Biol Chem. 2002;277(33):29832-9.

105. Cannon SB, Mitra A, Baumgarten A, Young ND, May G. The roles of segmental and tandem gene duplication in the evolution of large gene families in Arabidopsis thaliana. BMC Plant Biol. 2004:4:10

106. Schranz ME, Mitchell-Olds T. Independent ancient polyploidy events in the sister families Brassicaceae and Cleomaceae. Plant Cell. 2006;18(5):1152-65.

107. Town CD, Cheung F, Maiti R, Crabtree J, Haas BJ, Wortman JR, et al. Comparative genomics of Brassica oleracea and Arabidopsis thaliana reveal gene loss, fragmentation, and dispersal after polyploidy. Plant Cell. 2006; 18(6):1348-59.

108. Lou P, Wu J, Cheng F, Cressman LG, Wang X, McClung CR. Preferential retention of circadian clock genes during diploidization following whole genome triplication in Brassica rapa. Plant Cell. 2012;24(6):2415-26.

109. Larkin MA, Blackshields G, Brown NP, Chenna R, McGettigan PA, McWilliam H, et al. Clustal W and Clustal X version 2.0. Bioinformatics. 2007;23(21):2947-8.

110. Kumar S, Stecher G, Tamura K. MEGA7: molecular evolutionary genetics analysis version 7.0 for bigger datasets. Mol Biol Evol. 2016;33(7):1870-4.

\section{Publisher's Note}

Springer Nature remains neutral with regard to jurisdictional claims in published maps and institutional affiliations. 